\title{
An Easy and Summarized Approach to Integrated Management of Childhood Illness (IMCI)
}

Agrawal $k$., 米

\section{Introduction:}

Every year, about 10.5 million children die before their $5^{\text {th }}$ birthday in developing countries, out of which $70 \%$ of these are due to:

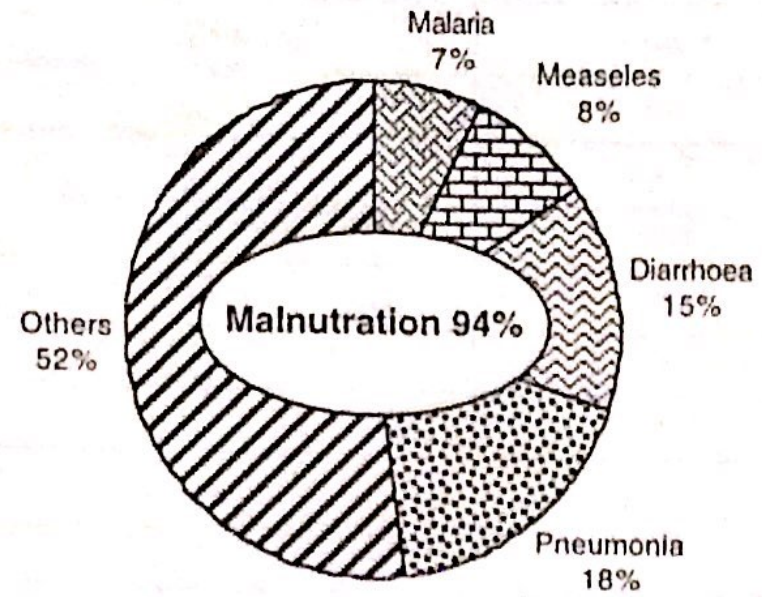

IMCI guidelines approach childhood illness in a systematic way, combining the steps needed to manage several different conditions, particularly ARI. Diarrhoca. Measles, Malaria and Malnutrition. Because of its systematic approach, it has now become a part of the MBBS and MD (Paediatric) course. This article is useful for general practice in field or hospital GOPD and is an easy and systematic approach to handle children less than 5 years of age wo come to the emergency and out patient department of or hospital.

\section{Objectives of IMCI:}

- Accurately assess, classify and treat sick children following $\mathrm{IMCl}$ guidelines.

- Correctly refer seriously ill patients after giving pre-referral treatment.

- Counsel care takers regarding home treatment follow up and when to return immediately.

- Routinely check immunization status.

:) Dr. Keshav Agrawal, MBBS. Instructor IMCI

Capl. Medical Officer

Shree Birendra Hospital, Chauni
- Carry out proper feeding assessments

- Appropriate nutrition and breast-feed counselling.

Complaints of $70 \%$ children attending paedia OPD are cough, loose motion, fever, ear prob! or decrease appetite.

IMCl provides a good, easy and systema approach to tackle these complaints.

(Note: - Assessment of fever in this article certain treatments modalities are not based on IM keeping in view of a hospital setting)

IMCI includes children aged from I week up t years, which is further divided in to 2 groups:

i. 1 week to $<2$ months

ii. 2 months to $<5$ years

Approach to a Child Aged 2 Months to . Years:

I. History + Assessment: should always inclu 6 points (which shall be elabora! sequentially)
A. GDS $\rightarrow$ General Danger Signs.
B. 4 main symptoms $\rightarrow$ cough / diarrhs / fever / ear problem.
C. Nutritional status of child.
D. Immunization status / Vitamin t Deworming
E. Feeding problem - if any.
F. Other problem.

\section{A. GDS $\rightarrow$ General Danger Signs:}

\section{Ask:}

i. Is the child able to drink or breastfeed?

ii. Does the child yomit every thing as soon as he/she consumes food?

iii. Has the child had convulsions during the period of illness. 
Look:

i. Is the child lethargic or unconscious. (If any of the above is present it suggests that the child has a GDS and is therefore serious).

B. 4 Main Symptoms $\rightarrow$ Cough / Diarrhoea / Fever / Ear Problem.

When the child presents with following symptoms, ask and look for the following signs, then classify and treat accordingly.

1. Cough or difficulty breathing:-

Ask:

- Duration of cough

Look:

- Respiratory rate (for full one minute when the child is silent)

- Subcostal indrawing.

- Stridor.

\begin{tabular}{|c|c|c|}
\hline Signs & Classify As & Treatment \\
\hline $\begin{array}{l}\text { - Any one GDS } \\
\text { or } \\
\text { kg) I/V stat and refer } \\
\text { or } \\
\text { - Stridor Present }\end{array}$ & $\begin{array}{l}\text { SEVERE PNEUMONIA } \\
\text { OR } \\
\text { Subcostal indrawing }\end{array}$ & $\begin{array}{l}\text { Inj. Ceftriaxone } \\
\text { VERY SEVERE DISEASE (@75mg/ }\end{array}$ \\
\hline $\begin{array}{l}\text { Fast breathing } \\
\text { ( } 2 \text { months }-<12 \text { months } \rightarrow \\
\geq 50 / \mathrm{min} \text {. } \\
(12 \text { months }-<5 \text { years } \rightarrow \\
\geq 40 / \mathrm{min} .\end{array}$ & PNEUMONIA & $\begin{array}{l}\text { Cortrimoxazole } \\
\text { (@ 6-8mg TMP/kg/day in } 2 \\
\text { divided doses } \times 5 \text { days) or } \\
\text { - Amoxycillin } \\
\text { (@ } 40-50 \mathrm{mg} / \mathrm{kg} / \text { day in } 3 \text { divided } \\
\text { doses } \times 5 \text { days } \\
\text { Paracetamol } \\
\text { (@ } 10 \mathrm{mg} / \mathrm{kg} / \text { dose } \times 8 \text { hourly) } \\
\text { - Salbutamol+Bromhexine syrup } \\
\text { (@.1-.2 } \mathrm{mg} / \mathrm{kg} / \text { dose } \\
\text { salbutamol } \times 8 \text { hourly } \\
\text { - Follow up after two days }\end{array}$ \\
\hline None of the above sign seen & COMMON COLD & $\begin{array}{l}\text { - Reassurance } \\
\text { - Soothing agent } \\
\text { - Follow up after five days }\end{array}$ \\
\hline
\end{tabular}

\section{Diarrhoea}

Ask

$\downarrow$ Duration of loose motion

+ Is blood present in stool or not

Look

- Alertness

- Lethargic/unconciousness

- Restlessness/irritable $\downarrow$ Sunken eye

$\downarrow$ Offer fluid

- Not able to drink

- Drinks eagerly (thirsty)

$\downarrow$ Skin pinch

- Very slow $\geq 2$ seconds

- Slow $<2$ seconds 


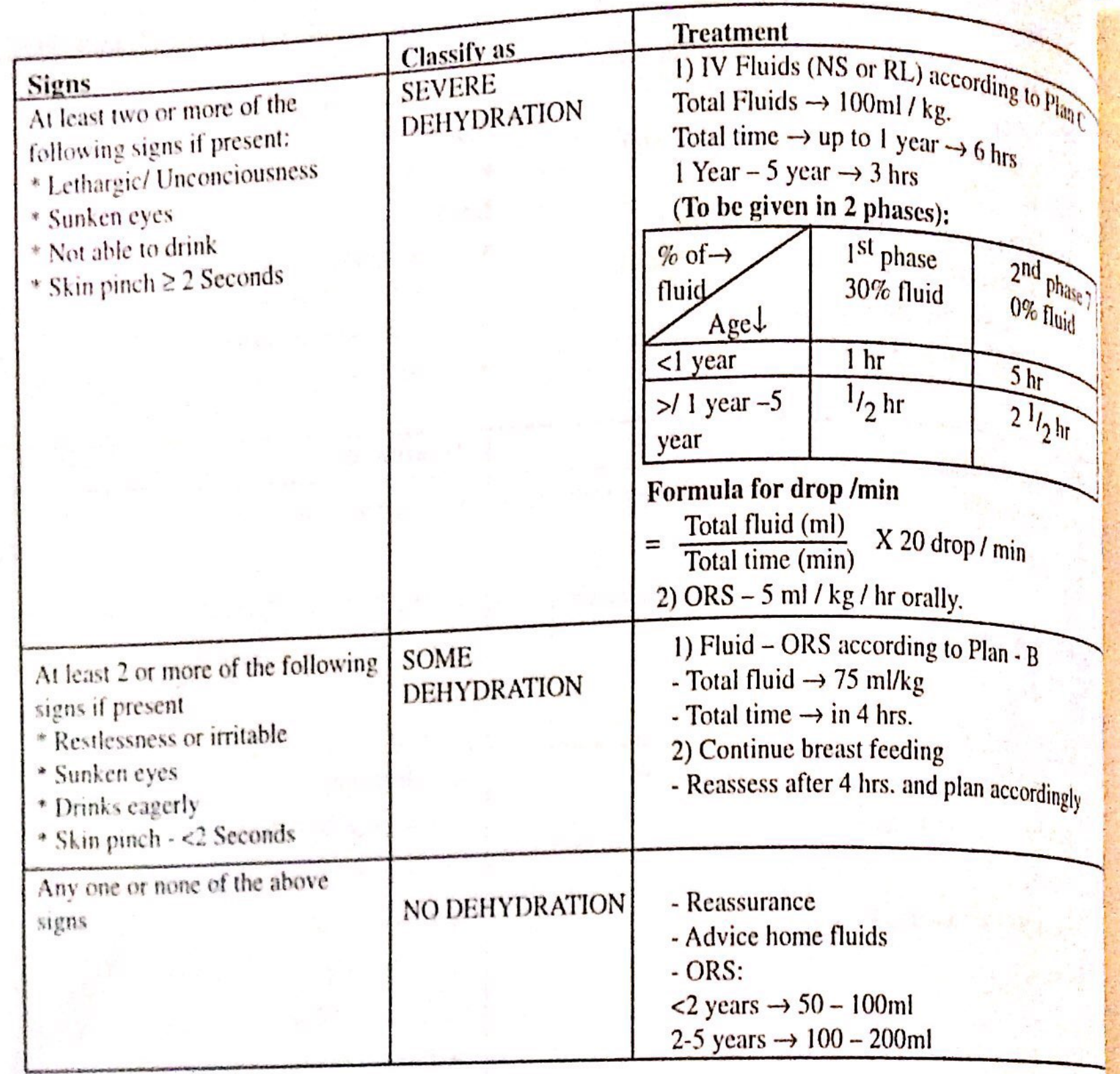

If diarrhoea $\geq 14$ days

\begin{tabular}{|c|c|c|}
\hline Signs & Classify as & Treatment \\
\hline $\begin{array}{l}\text { Some of severe dehydration if } \\
\text { present }\end{array}$ & $\begin{array}{l}\text { SEVERE } \\
\text { PERSISTENT } \\
\text { DIARRHOEA }\end{array}$ & $\begin{array}{l}\text { * Correct dehydration accordingly } \\
\text { * Vitamin A (If not given within } 6 \text { months) } \\
\text { * To avoid other forms of milk beside breast feeding } \\
\text { * Feeding advice (acc. to age discussed later) }\end{array}$ \\
\hline $\begin{array}{l}\text { No dehydration seen } \\
\text { - Feeding ativice } \\
\end{array}$ & $\begin{array}{l}\text { PERSISTENT } \\
\text { DIARRHOEA } \\
\end{array}$ & $\begin{array}{l}\text { *Vitamin A } \\
\text { * To avoid other forms of milk. }\end{array}$ \\
\hline If blood is present in stool & DYSENTRY & $\begin{array}{l}\text { * Correct dehydration accordingly. } \\
\text { * Cotrimoxazole } \\
(6-8 \mathrm{mg} / \mathrm{TMP} / \mathrm{kg} / \text { day in } 2 \text { divided dose } \times 5 \\
\text { days) } \\
\text { Nalidixic acid ( } 50 \mathrm{mg} / \mathrm{kg} / \text { day in } 4 \text { divided } \\
\text { dose } \times 5 \text { days) } \\
\text { Or } \\
\text { Nalidixic acid ( } 50 \mathrm{mg} / \mathrm{kg} / \text { day in } 4 \text { divided } \\
\text { dose } \times 5 \text { days) } \\
\qquad \downarrow \\
\text { Follow up after } 2 \text { days. (if no improvement seen) } \\
\text { Metronidazole ( } 40 \mathrm{mg} / \mathrm{kg} / \text { day in } 3 \text { divided dose } 5 \text { day) }\end{array}$ \\
\hline
\end{tabular}




\section{Fever:}

(not based on $\mathrm{IMCI}$ ), keeping in view that Kathmandu falls in a non malarial region) includes common causes of fever seen in children.

\begin{tabular}{|c|c|c|}
\hline Signs & Classify as & Treatment \\
\hline * Convulsions & $\begin{array}{l}\text { FEBRILE } \\
\text { CONVULSION }\end{array}$ & $\begin{array}{l}\text { * Reassurance } \\
\text { * Paracetamol } \\
(10 \mathrm{mg} / \mathrm{kg} / \text { dose } \times \mathrm{SOS}) \text { and refer }\end{array}$ \\
\hline $\begin{array}{l}\text { * Headache } \\
\text { * Stiff Neck } \\
\text { * Headache } \\
\text { * Coated tongue } \\
\text { *Abdomen pain } \\
\text { * Spleenomegaly }\end{array}$ & $\begin{array}{l}\text { MENINGITIS } \\
\text { ENTERIC } \\
\text { FEVER }\end{array}$ & $\begin{array}{l}\text { * Amoxycillin } \\
(100 \mathrm{mg} / \mathrm{kg} / \text { day in } 3 \text { divided dose }) \text { and refer } \\
\text { * Ofloxacin } \\
10-15 \mathrm{mg} / \mathrm{kg} / \text { day in } 2 \text { divided dose } \times 7 \text { days } \\
\text { Or } \\
\text { * Cotrimoxazole } \\
(8 \mathrm{mg} / \mathrm{kg} / \text { day TMP in } 2 \text { divided dose } \times 14 \text { days }) \\
\text { * Paracetamol }\end{array}$ \\
\hline $\begin{array}{l}\text { * Macular Rash } \\
\text { * Running Nose or } \\
\text { * Cough or } \\
\text { * Red Conjunctiva }\end{array}$ & MEASELES & $\begin{array}{l}* \text { Vitamin A On day } 0 \text { and day } 1 \\
(6-12 \text { months } \rightarrow 1 \text { lakh unit } \\
12 \text { months }-5 \text { yrs } \rightarrow 2 \text { Lakh Unit })\end{array}$ \\
\hline $\begin{array}{l}\text { * Headache } \\
\text { * Pallor } \pm \\
\text { * History of travel to malarious } \\
\text { region within } 30 \text { days. }\end{array}$ & MALARIA & $\begin{array}{l}\text { Syp. Chloroquine } \\
10 \mathrm{mg} / \mathrm{kg} \text { stat } \\
5 \mathrm{mg} / \mathrm{kg} \text { after } 6 \mathrm{hrs} \\
5 \mathrm{mg} / \mathrm{kg} \times \mathrm{BD} \times 2 \text { days }\end{array}$ \\
\hline $\begin{array}{l}\text { * Pustular rash present all over } \\
\text { body }\end{array}$ & PYODERMA & $\begin{array}{l}\text { * Cloxacillin } \\
50 \mathrm{mg} / \mathrm{kg} / \text { day in } 4 \text { divided dose } \times 5 \text { days. }\end{array}$ \\
\hline $\begin{array}{l}\text { *Abdominal Pain } \\
{ }^{*} \text { Burning micturation (cries } \\
\text { during micturation } \\
{ }^{*} \pm \text { Hematuria } \\
{ }^{*} \text { Vomiting }\end{array}$ & UTI & $\begin{array}{l}* \text { Cotrimoxazole } 8 \mathrm{mg} \text { of TMP } / \mathrm{kg} / \text { day } \times \mathrm{BD} \times \\
5 \text { days } \\
* \text { Norfloxacin } 10 \mathrm{mg} / \mathrm{kg} / \text { day } \times \mathrm{BD} \text { in } 2 \text { divided } \\
\text { doses } \times 5 \text { days }\end{array}$ \\
\hline $\begin{array}{l}{ }^{*} \text { Headache } \\
\text { * Sinus tenderness } \\
\text { * Blocked nose }\end{array}$ & SINUSITIS & $\begin{array}{l}\text { *Amoxycillin } \\
(40-50 \mathrm{mg} / \mathrm{kg} / \text { day in } 3 \text { divided doses } \times 5 \text { days) } \\
\text { Or } \\
{ }^{*} \text { Erythromycin } \\
30 \mathrm{mg} / \mathrm{kg} / \text { day in } 4 \text { divided doses } \times 5 \text { days } \\
{ }^{*} \text { Normal Saline Drops intra-nasally } \times \text { TDS }\end{array}$ \\
\hline $\begin{array}{l}\text { * Throat Pain } \\
{ }^{*} \text { Congested and enlarged tonsils }\end{array}$ & TONSILLITIS & As above. \\
\hline
\end{tabular}

\section{Ear Problem:}

Ask:

$\downarrow \quad$ Is Ear pain present?

$\downarrow \quad$ Is there ear discharge?

$\downarrow$ If present-duration

Look:

$4 \quad$ Pus draining from ear

$\downarrow$ Tender swelling behind ear 


\begin{tabular}{|c|c|c|}
\hline Signs & Classify as & Treatment \\
\hline $\begin{array}{l}\text { Tender Swelling behind ear } \\
\text { * Ear pain present or } \\
\text { * Pus Discharge present }<14 \\
\text { days }\end{array}$ & $\begin{array}{l}\text { MASTOIDITIS } \\
\text { ACUTE EAR } \\
\text { INFECTION }\end{array}$ & $\begin{array}{l}\text { Cotrimoxazole/Amoxycillin stat dose and reft } \\
* \text { Cotrimoxazole } 8 \mathrm{mg} \mathrm{TMP} / \mathrm{kg} / \text { day in two } \\
\text { divided dose } \times 5 \text { day or } \\
\text { * Amoxycillin } \\
\text { High dose } 70-90 \mathrm{mg} / \mathrm{kg} / \text { day in } 3 \text { divided do } \\
\times 10 \text { days } \\
* \text { Paracetamol ( } 10 \mathrm{mg} / \mathrm{kg} / \mathrm{dose}) \\
\text { * Dry wicking to be done. }\end{array}$ \\
\hline - Pus Discharge $\geq 14$ days & $\begin{array}{l}\text { CHRONIC EAR } \\
\text { INFECTION }\end{array}$ & Dry wicking and refer. \\
\hline No pain or discharge & $\begin{array}{l}\text { NOEAR } \\
\text { INFECTION }\end{array}$ & $\begin{array}{l}\text { Symptomatic treatment } \\
\text { Or } \\
\text { Refer. }\end{array}$ \\
\hline
\end{tabular}

\section{Malnutrition and Anaemia}

Look and feel:

- Visible severe wasting

- edema of both feet

- Palmar pallor

- Severe pallor

- some pallor

* Determine weight for age (chart given)

\begin{tabular}{|l|l|l}
\hline Signs & Classify as & Treatment \\
\hline $\begin{array}{l}\text { Severe visible wasting or } \\
\text { bilaterat pedal edema or } \\
\text { Severe pallor }\end{array}$ & $\begin{array}{l}\text { SEVERE MALNUTRITION } \\
\text { OR } \\
\text { SEVERE ANAEMIA }\end{array}$ & $\begin{array}{l}\text { Vitamin A<12 months - 1 lakh } \\
\text { unit } \\
12 \text { months }-5 \text { year }-2 \text { lakh unit } \\
\text { and refer }\end{array}$ \\
\hline $\begin{array}{l}\text { Some palmar pallor or } \\
\text { lery low weight for age }\end{array}$ & ANAEMIA & $\begin{array}{l}\text { Feeding advice (discussed later) } \\
\text { If age }>2 \text { years } \\
\text { Olbendazole (400mg) stat } \\
\text { If pallor present Iron } \\
\text { Gmg/kg/dose } x \text { BD } \times 14 \text { days } \\
\text { Folow up aftrer 14 days }\end{array}$ \\
\hline None of the above signs & NO ANAEMIA & $\begin{array}{l}\text { No treatment } \\
\text { If age <2 years - give feeding } \\
\text { advice }\end{array}$ \\
\hline
\end{tabular}

\section{Immunization Status (National Immunization Schedule)}

Birth or first visit:

6 weeks age or 6 weeks after BCG:

10 weeks age or 1 month after ${ }^{\text {st }}$ dose:

14 weeks or 2 month after $1^{\text {st }}$ dose:

9 months age or:

Thereafter on first presentation
BCG, OPV

DPT-1, OPV-1

DPT-2, OPV-2

DPT-3, OPV-3

Measeles 
- Vitamin A Supplementation

- upto 6 months $\times$

- 6 months to $<12$ months

_ cap $(1,00,000$ unit $)$

- 12 months to 5 years

1 cap $(2,00,000$ unit $)$

- Vitamin A Supplementation should be done 6 monthly upto 5 years.

- Vitamin A as treatment is given for

- Measles

on day 0 , day 1

- Malnutrition on day 0 , and 1

- Night blindness on day $0,1 \& 21$

\section{Deworming}

From the age of 2 years, every 6 monthly deworming should be done with Albendazole (400mg) up to the age of 5 years.

\section{E. Feeding Advice}

(a) From birth to 6 months:

- Exclusive breastfeeding, at least 8 times $/ 24$ hrs.

- Discourage use of any other fluid/food or artificial foods

- Breastfeeding should be al least for 10 minute per sitting.

(b) 6 to 12 months

- Breastfeeding any time as for baby's want.

- Introduce food items like rice, dal, jaulo, haluwa, milk + sugar+roti, fruits etc.

- The above items should be taken at least 3 times a day for breast fed babies and $S$ times a day for not breastfed babies.

(c) 12 months to 2 years

Cominue breastfeed as per demand.

the above mentioned food/food cooked for other member of family should be given at least 5 times irrespective of breastfeed.

(d) 2 years and above

Food cooked for other members should be given at least 3 times a day.

Following iterns should be given at least 2 times/day (Chiura cooked in ghee/oil, fruits, maize, pulses and other proteineous food)

Advice about breastfecding

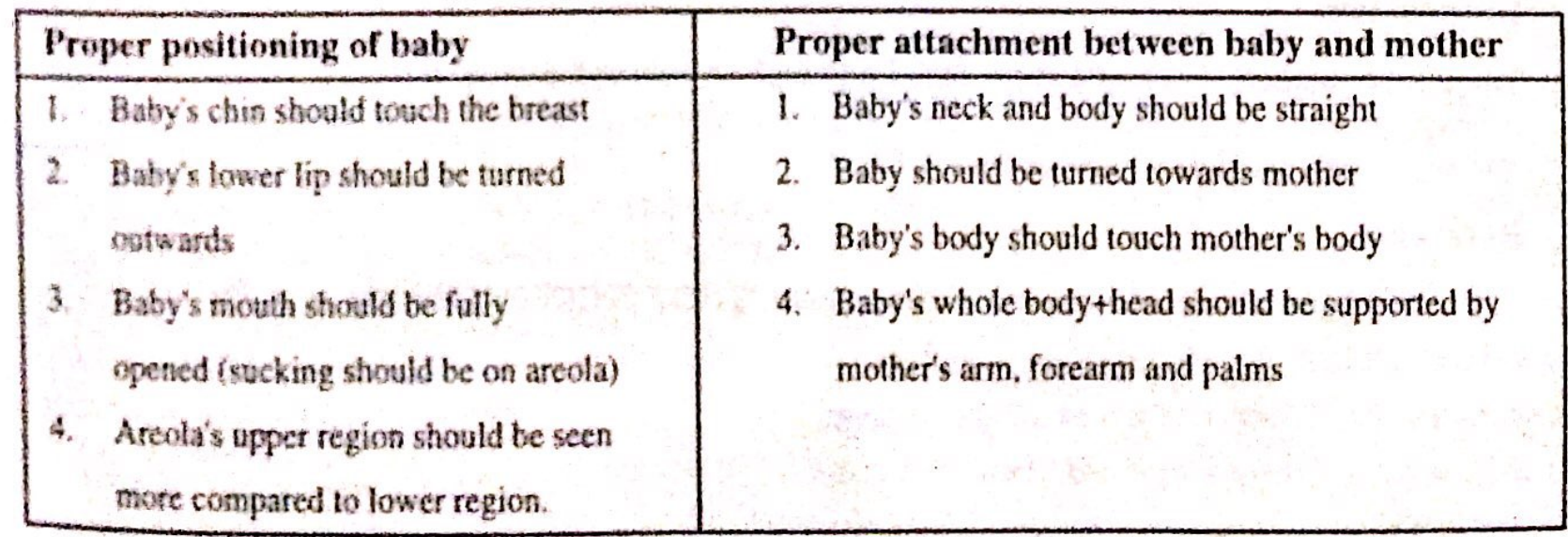




\section{F. Other Problem:}

This category includes complaints other than mentioned earlier, which can be managed based, personal knowledge and exposure or life.

\section{Approach to a Child (1 Week to 2 Months)}

1. Ask and look for signs for any serious bacterial infection:

\begin{tabular}{|c|c|c|}
\hline Ask/look/see/feel & Classify as & Treatment \\
\hline $\begin{array}{l}\text { - H/O convulsion or } \\
\text { - RR }>60 / m i n \text { or } \\
\text { - Severe chest indrawing or } \\
\text { - Nasal naring or } \\
\text { - Grunting or } \\
\text { - Bulging fontanelle or } \\
\text { - Ear discharge or } \\
\text { - Pus discharge from umbilical region } \\
\text { - Fith inflammation spread to skin or } \\
\text { - Fever (s } 37.50 \text { F or } \\
\text { - Lethargie/Unconscious or } \\
\text { - Movement of limbs less than normal or } \\
\text { - Severe of many pustular rash present over skin or } \\
\text { - Unable to suck or } \\
\text { - Yellow dicoloration of body or } \\
\text { - Blarsh discoloration of extremities and lip }\end{array}$ & $\begin{array}{l}\text { SEVERE } \\
\text { BACTERIAL } \\
\text { INIECTION }\end{array}$ & $\begin{array}{l}\text { Inj PPF } \\
(50,000 \mathrm{IU} / \mathrm{kg} \text { stat } \mathrm{I} / \mathrm{M} \text { ) } \\
+ \\
\text { Inj. Gentamycin } \\
(2.5 \mathrm{mg} / \mathrm{kg} \mathrm{I} / \mathrm{M} \\
\text { stat }) \text { and refer }\end{array}$ \\
\hline $\begin{array}{l}\text { - Timbihcus ted and pus discharge with no } \\
\text { involvement of skin or } \\
\text { - Few pustules over skin }\end{array}$ & $\begin{array}{l}\text { LOCAL } \\
\text { BACTERIAL } \\
\text { INFECTION }\end{array}$ & $\begin{array}{l}\text { Amoxycillin } \\
40-50 \mathrm{mg} / \mathrm{kg} / \text { day } \\
\text { in } 3 \text { div doses } \times 5 \text { days } \\
\text { Cleaning with antibioti } \\
\text { solution }\end{array}$ \\
\hline
\end{tabular}

\section{Diarrhoea:}

- Simitar to assessment done for age group

- 2 months -5 years, except that we do not ascess thirst.

- Classification and treatment are similar to the other age group.

\section{Conclusion:}

The above method was taken from IMCI although certain symptoms, sign and various treatme straterges were modified keeping in view of our hospital setting. This will surely help a lot in examini children in the peripheries and in the GOPD.

\section{Reference:}

1. IMCl guidelines (His majesty government / WHO / UNICEF / USAID) 